\title{
Argentine validation of the Consideration of Future Consequences Scale (CFC-14)
}

\author{
Ignacio Acuña; Mauricio Federico Zalazar-Jaime; Yanina Michelini; \\ Juan Ignacio Guzmán; Juan Carlos Godoy; Ezequiel Galarce; Jeffrey Joireman
}

How to cite this article:

Acuña, I., Zalazar-Jaime, M.F., Michelini, Y., Guzmán, J.I., Godoy, J.C., Galarce, E. \& Joireman, J. (2020). Argentine validation of the Consideration of Future Consequences Scale (CFC-14): Acta Colombiana de Psicología, 23(1), $357-368$. doi: http://www.doi.org/10.14718/ACP.2020.23.1.16

Recibido, marzo 21/2018; Concepto de evaluación, abril 27/2019; Aceptado, marzo 6/2019

\author{
Ignacio Acuña \\ ORCID: https://orcid.org/0000-0001-6152-6530 \\ Universidad Nacional de Córdoba, Córdoba, Argentina \\ Mauricio Federico Zalazar-Jaime \\ ORCID: https://orcid.org/0000-0001-5793-9770 \\ Universidad Nacional de Córdoba, Córdoba, Argentina \\ Yanina Michelini \\ ORCID: https://orcid.org/0000-0003-0768-7645 \\ Universidad Nacional de Córdoba, Córdoba, Argentina \\ Juan Ignacio Guzmán \\ ORCID: https://orcid.org/0000-0002-9996-6694 \\ Universidad Nacional de Córdoba, Córdoba, Argentina \\ Juan Carlos Godoy \\ ORCID: https://orcid.org/0000-0002-1622-1647 \\ Universidad Nacional de Córdoba, Córdoba, Argentina \\ Ezequiel Galarce \\ ORCID: https://orcid.org/0000-0002-6302-4523 \\ Rally Health, San Francisco, Estados Unidos \\ Jeffrey Joireman \\ ORCID: https://orcid.org/0000-0002-6102-3420 \\ Washington State University, Washington, Estados Unidos
}

\begin{abstract}
Consideration of future consequences is the extent to which people anticipate and are influenced by the potential future consequences of their current behavior. A well-established tool to measure this behavior is the 14-item Consideration of Future Consequences Scale (CFC-14). The CFC-14 has shown appropriate psychometric properties in several languages. This scale comprises two factors: the CFC-Immediate (CFC-I, 7 items) and the CFC-Future (CFC-F, 7 items). The main goal of this study was to assess the psychometric properties and internal consistency of the CFC-14 Scale in Spanish, using an Argentine sample. A second goal was to determine its convergent validity with impulsivity, and determine differences and invariance across gender and age groups. Using a web-based survey, data were collected from 512 participants $(75.2 \%$ women) aged 13-74 years $(\mathrm{M}=30.8)$. CFA showed a two-factor model as the best solution for the 13-items version (CFI .961 , TLI .952, RMSEA .064 90\%IC .054/.074, WRMR 0.979). Standardized regression weights ( $\mathrm{p} \leq .05)$ ranged from .50 to .66 for CFC-F and between .43 and .83 for CFC-I. Composite reliability was also adequate: CFC-F achieved $\rho=.80$ and CFC-I $\rho=.82$. There were no differences across gender and age, but there was a progressive invariance between these groups. The CFC-F and UPPS-P subscales correlations were negative and significant, highlighting the negative and moderate correlation between CFC-F and the lack of premeditation $(r=-.41)$. Thus, CFC-14 has adequate psychometric properties in an Argentine population, although more studies are necessary to determine the robustness of these findings.

Key words: consideration of future consequences, Argentine population, impulsivity, psychometric properties, confirmatory factor analysis.
\end{abstract}

* IIPsi-UNC-CONICET, Facultad de Psicología, Universidad Nacional de Córdoba, Bv. de la Reforma esq. Enfermera Gordillo s/n, Ciudad Universitaria, Córdoba, C. P.: 5000, Argentina. ignacio.acuna@unc.edu.ar 


\title{
Validación argentina de la escala de Consideración de las Consecuencias Futuras (CFC-14)
}

\author{
Resumen
}

\begin{abstract}
La consideración de las consecuencias futuras se define como el grado en el cual las personas anticipan y son influenciadas por las potenciales consecuencias futuras de su comportamiento actual, y una herramienta muy utilizada para medirla es la Escala de Consideración de las Consecuencias Futuras (CFC-14). Esta escala ha exhibido propiedades psicométricas adecuadas en varios idiomas y se encuentra conformada por dos factores: CFC-Inmediato (CFC-I, 7 ítems) y CFC-Futuro (CFC-F, 7 ítems). El objetivo principal de este estudio fue evaluar las propiedades psicométricas y la consistencia interna de la versión en español de la CFC-14 en una muestra argentina, además de identificar las evidencias de validez convergente con la Escala de Impulsividad y la invarianza en función del género y la edad de los participantes. Para esto, se evaluó mediante una encuesta online a 512 participantes $(75.2 \%$ mujeres $)$ de 13 a 74 años $(\mathrm{M}=30.8)$ y se realizó un $\mathrm{AFC}$ en el que se encontró un modelo de dos factores como aquel con mejor ajuste para una versión de la escala de 13 ítems (CFI = .961; TLI = .952; RMSEA =.064; IC $90 \%=.054-.074 ;$ WRMR = 0.979). Específicamente, los pesos de regresión estandarizados $(\mathrm{p} \leq .05)$ fueron de .50 a .66 para CFC-F y de .43 a .83 para CFC-I; los valores de confiabilidad compuesta fueron adecuados, con un $\rho=.80$ para CFC-F y un $\rho=.82$ para CFC-I; no se observaron diferencias estadísticamente significativas en función del sexo y la edad de los participantes, no obstante, hubo una invarianza progresiva entre estos grupos; y las correlaciones entre las subescalas de la Escala de Impulsividad (UPPPS-P) y la CFC-F fueron negativas y significativas, siendo llamativa la correlación negativa y moderada entre la falta de premeditación y la CFC-F $(r=-.41)$. De este modo, la CFC-14 mostró propiedades psicométricas adecuadas en una muestra argentina, aunque se necesita de más estudios para determinar la robustez de estos resultados.

Palabras clave: consideración de las consecuencias futuras, población argentina, impulsividad, propiedades psicométricas, análisis factorial confirmatorio.
\end{abstract}

\section{Introducción}

A diario, las personas toman decisiones que influyen sobre su futuro tanto inmediato como lejano. Por ejemplo, que una persona limite el consumo de grasas en una dieta podría mejorar su salud a largo plazo, aunque a corto plazo su vida podría ser menos placentera; de manera similar, cuando un estudiante decide estudiar durante el fin de semana para obtener mejores calificaciones, es probable que con ello resigne momentos de diversión con sus amigos. Estas decisiones pueden tomarse con respecto a cuestiones triviales, como elegir entre un jugo de naranja o uno de manzana, pero también pueden ser respecto a cuestiones que pueden impactar el curso de la vida de alguien, como decidir sobre una carrera universitaria, por ejemplo (Nigro, Cosenza, Ciccarelli \& Joireman, 2016); de este modo, en la elección de las personas la consideración de las consecuencias futuras (CFC) tiene un rol importante tanto para sus objetivos a corto como a largo plazo, si se entiende la CFC como el grado en el cual las personas anticipan y son influenciadas por las potenciales consecuencias futuras de su comportamiento actual (Joireman \& King, 2016; Strathman, Gleicher, Boninger \& Edwards, 1994).

En investigaciones recientes, la CFC ha sido asociada con al menos cuatro constructos: con los comportamientos saludables, las conductas de riesgo y el rendimiento académico, con la agresión, con el comportamiento organizacional prosocial, y con las conductas y actitudes pro-ambientales (Joireman \& King, 2016; Joireman, Strathman \& Balliet,
2006); e, incluso, varios estudios indican que mayores puntuaciones en CFC se relacionan con un incremento en la satisfacción con la vida (Azizli, Atkinson, Baughman \& Giammarco, 2015) y el optimismo (Geers, Wellman, Seligman, Wuyek \& Neff, 2010).

Teniendo esto en cuenta, y reconociendo la importancia de la CFC en la toma de decisiones, Strathman et al. (1994) desarrollaron una escala para evaluarla, y en el análisis de su consistencia y estructura interna, incluido un test-retest y análisis factoriales exploratorios y confirmatorios, la escala obtuvo una estructura unidimensional compuesta por una variable latente y 12 ítems. Sin embargo, en otros estudios, a pesar de presentar propiedades psicométricas adecuadas, aún no existe consenso respecto a su estructura factorial (Bruderer-Enzler, 2015; Joireman \& King, 2016; Toepoel, 2010), y en algunos de ellos se afirma que un modelo compuesto por dos factores sería más apropiado para ella que el modelo de un factor propuesto originalmente (Adams, 2012; Bruderer-Enzler, 2015; Joireman, Balliet, Sprott, Spangenberg \& Schultz, 2008; Petrocelli, 2003; Toepoel, 2010). Incluso, algunos estudios indican que una estructura de dos factores se ajusta mejor a los datos, además de que permite explicar la relación entre la CFC como constructo psicológico y otras características conductuales y psicológicas como el autocontrol o el descuento temporal (Joireman et al., 2008).

En dicho modelo de dos factores, el primer factor se ha relacionado con la consideración de las consecuencias futuras (CFC-F), en la que se ubicarían cinco ítems, con 
ítems como "considero cómo serán los eventos en el futuro $\mathrm{y}$ trato de influenciar esos eventos con mi comportamiento diario"; mientras que el segundo factor reflejaría la consideración de las consecuencias inmediatas (CFC-I), en la que se ubicarían siete ítems, con ítems como "solo actúo para satisfacer mis preocupaciones inmediatas, pienso que en el futuro se resolverán solas" (Strathman et al., 1994).

En una reciente actualización de la escala, Joireman Shaffer, Balliet y Strathman (2012) agregaron dos ítems a la subescala CFC-F con el fin de equiparar el número ítems por factor, con lo que se obtuvo como resultado la versión CFC-14. Con esta actualización, Joireman et al. (2012) reportaron que el modelo de dos factores se ajustó mejor que el modelo de un factor, con pesos de regresión estandarizados de .48 a .75 para la CFC-F y de .53 a .86 para la CFC-I, y su confiabilidad - medida con el coeficiente alfa de Cronbach - fue, asimismo, satisfactoria para ambas subescalas ( $\alpha=.84$ para la CFC-I y $\alpha=.80$ para la CFC-F).

Posteriormente, se encontraron resultados similares en estudios llevados a cabo en diferentes poblaciones, como ocurre con el estudio de Nigro et al. (2016), en población general italiana, en el que se evaluó una gran muestra de participantes de ambos sexos de 16 a 75 años de edad; o con el de Camus, Berjot y Ernst-Vintila (2014), realizado en población francesa, aunque en este se obtuvo evidencia de la estructura de dos factores en una muestra más reducida, conformada por estudiantes universitarios de 17 a 37 años. En ninguno de estos dos estudios se buscó evidencia de la validez convergente del instrumento.

Ahora bien, a pesar de que la estructura de dos factores con siete ítems por factor exhibió propiedades psicométricas adecuadas, algunas adaptaciones respaldan el uso de la versión de 12 ítems. Por ejemplo, Vásquez-Echeverría et al. (2015) observaron, contrario a Strathman et al. (1994), que una estructura de dos factores para la versión de 12 ítems se ajustó mejor a los datos en una muestra de estudiantes universitarios portuguesa; no obstante, los índices de confiabilidad fueron similares a los reportados en estudios previos, pues indicaron un valor por debajo de lo esperado para el factor CFC-F (CFC-F: $\alpha=.58$; CFC-I: $\alpha=.82$ ). Sin embargo, en otro estudio (Vásquez-Echeverría, Martín, Esteves, Ortuño \& Joireman, 2017), se replicó el procedimiento de validación de la versión de 12 ítems con una muestra de jóvenes uruguayos, y encontraron que el modelo de dos factores presentaba un mejor ajuste que el modelo de un factor. De modo que los resultados se encuentran aún sin un claro consenso.

Por otra parte, además de analizar las propiedades psicométricas de la escala, también es importante estudiar la relación de la CFC con otros rasgos y variables sociodemográficas, sin embargo, e igual que con el punto anterior, con respecto a las diferencias por sexo, por ejemplo, la literatura no presenta resultados unívocos. Así, mientras que algunos estudios reportan que hombres y mujeres no difieren significativamente en las puntuaciones en la CFC (Nigro et al., 2016), otros indican que los hombres puntúan más alto en la subescala CFC-I que las mujeres (Camus et al., 2014; Vásquez-Echeverría et al., 2015, 2017), lo cual, a su vez, indicaría que las mujeres presentan mayor consideración de las consecuencias futuras (Camus et al., 2014).

Finalmente, aunque varios estudios han reportado adaptaciones internacionales de la CFC (Camus et al., 2014; Nigro et al., 2016), según los autores del presente trabajo existen solo dos en Iberoamérica, el de Vásquez-Echeverría et al. (2015) para la población portuguesa y el de VásquezEcheverría et al. (2017) para la población uruguaya, por lo que, interesantemente, no se han encontrado estudios de este tipo con población argentina.

Ahora bien, considerando las diferencias culturales y del uso del lenguaje en Argentina y otros países donde la escala CFC se ha adaptado, resulta importante y útil estudiar de qué manera la $\mathrm{CFC}$, como constructo psicológico, funciona en la población de este país, además de cómo, en un futuro cercano, se relaciona con el comportamiento de riesgo y saludable. En este contexto, el presente estudio tuvo como objetivo analizar la estructura factorial, la confiabilidad y la validez de la escala Consideración de las Consecuencias Futuras de 14 ítems (CFC-14), de Joireman et al. (2012) en una muestra de adolescentes, jóvenes y adultos de Córdoba, una ciudad ubicada en el centro de Argentina. Adicionalmente, se analizaron evidencias de validez convergente de la escala con el constructo de impulsividad, haciendo comparaciones con la Escala de Impulsividad (UPPS-P), la cual presenta propiedades psicométricas adecuadas en la población local (Pilatti, Lozano \& Cyders, 2015). Finalmente, se exploró si el patrón de respuestas a la escala difirió en función del sexo y la edad de los participantes.

\section{Método}

\section{Participantes}

La muestra estuvo conformada por 510 participantes de ambos sexos (75.2 \% mujeres) de 13 a 74 años de edad $(\mathrm{M}=30.77, \mathrm{SD}=10.06)$. Los participantes, todos hispanohablantes, fueron reclutados por medio de redes sociales -Facebook o Twitter, por ejemplo-y listas de correo electrónico, y no recibieron ninguna compensación por su participación. Debido al proceso de reclutamiento de tipo online y la falta de compensaciones por la participación, no fue posible obtener una muestra balanceada según el 
sexo de los participantes. Con respecto al nivel educativo, el $35 \%$ de la muestra presentaba terciario o universidad incompletos, el $32.8 \%$ tenía algún estudio de posgrado completo o incompleto, el $25.1 \%$ había finalizado el terciario o universidad, el $4.7 \%$ completó el secundario y el $2 \%$ reportó secundario incompleto.

\section{Diseño}

Este estudio se basó en un diseño instrumental para adaptar la escala de CFC a la población argentina y explorar su estructura factorial, además de su confiabilidad (Montero \& León, 2007).

\section{Proceso de traducción de los items de la escala CFC-14}

Cuatro expertos, competentes en el idioma inglés y en evaluación psicológica, realizaron la traducción directa de la escala original de CFC-14. El primer autor de este artículo comparó y compiló las diferentes versiones, realizando los ajustes idiomáticos pertinentes; proceso con el que buscó mantener la equivalencia conceptual —que los ítems originales y traducidos midan el mismo constructo-, semántica -que la redacción realizada en la versión traducida del ítem tenga el mismo significado que el texto del ítem original-y funcional de la escala - que las acciones descritas en ambas versiones de los ítems presenten objetivos y dificultades similares en ambas culturas-(Mimura \& Griffiths, 2008). Considerando estas equivalencias, todos los ítems fueron traducidos sin realizar modificaciones adicionales.

\section{Instrumentos}

Escala de Consideración de las Consecuencias Futuras (CFC-14; Joireman et al., 2012). Esta escala está compuesta por 14 ítems que evalúan las consecuencias del comportamiento distantes (CFC-F, siete ítems) y próximas (CFC-I, siete ítems) en el tiempo. Los participantes deben responder a los diferentes enunciados (por ejemplo, "estoy dispuesto/a a sacrificar mi felicidad o bienestar inmediato para lograr resultados futuros") mediante una escala tipo Likert con siete opciones de respuesta, donde 1 es "no me representa para nada" y 7 , "me representa totalmente". Respecto a las propiedades psicométricas, este instrumento posee estudios de estructura y consistencia interna con resultados satisfactorios (alfas de Cronbach $>.80$ para ambas subescalas).

Escala de Impulsividad (UPPS-P; Verdejo-García, Lozano, Moya, Alcázar \& Pérez-García, 2010). La versión de la escala utilizada fue adaptada por Pilatti et al. (2015) y está compuesta por 59 ítems que evalúan cinco dimensiones de la personalidad impulsiva, incluidas la urgencia negativa - con ítems como "tengo problemas para resistir mis antojos [de comida, cigarrillos, etc.]"-, la falta de perseverancia — con ítems como "tiendo a rendirme fácilmente"-, la falta de premeditación — con ítems como "no soy una de esas personas que sueltan las cosas sin pensar"-, la búsqueda de sensaciones — con ítems como "generalmente busco experiencias y sensaciones nuevas y excitantes" - y la urgencia positiva - con ítems como "cuando estoy muy contento, tiendo a hacer cosas que pueden causarme problemas en mi vida" - Los participantes deben responder a cada enunciado haciendo uso de una escala tipo Likert con cuatro opciones de respuesta, donde 1 significa estar "muy de acuerdo" y 4 , "muy en desacuerdo". Respecto a sus propiedades psicométricas, la versión implementada presentó valores de confiabilidad adecuados para todas las subescalas: urgencia negativa $(\alpha=.82)$, falta de premeditación $(\alpha=.83)$, falta de perseverancia $(\alpha=.74)$, búsqueda de sensaciones $(\alpha=.86)$ y urgencia positiva $(\alpha=.93)$ (Pilatti et al., 2015; Pilatti, Rivarola Montejano, Lozano \& Pautassi, 2016).

\section{Procedimiento}

La recolección de datos se llevó a cabo durante el mes de febrero de 2016. Antes de completar el cuestionario, los participantes recibieron información sobre el objetivo del estudio y dieron su consentimiento informado; después, al momento de contestar las preguntas, recibieron avisos electrónicos por cada respuesta faltante, esto con el fin de minimizar la probabilidad de obtener datos incompletos. En total, completar el cuestionario les llevó, aproximadamente, 20 minutos.

\section{Análisis de datos}

Inicialmente, se calculó la media, la desviación estándar, la asimetría y la curtosis de los datos obtenidos. Específicamente, como criterio para evaluar los índices de asimetría y curtosis se consideró como excelentes los valores \pm 1.00 y adecuados los valores \pm 2.00 (George \& Mallery, 2011); también, se identificaron los casos atípicos univariados mediante el cálculo de puntuaciones $\mathrm{Z}$ - puntuaciones $\mathrm{z}> \pm 3.29$ fueron considerados atípicos- $-\mathrm{y}$ multivariados mediante la prueba de distancia de Mahalanobis $(\mathrm{p}<.001)$; y se evaluó el patrón de los valores perdidos con el fin de estimar si el mismo respondía a una distribución aleatoria; todo por medio del IBM-SPSS, v. 19.0 (Tabachnick \& Fidell, 2007).

Después, se realizó un análisis factorial confirmatorio (AFC) con el fin de evaluar la estructura interna de la escala CFC-14, para lo cual se utilizó el software estadístico Mplus, versión 6.12, ya que permite aplicar el método de estimación 
de mínimos cuadrados ponderados (WLS), considerado como el más adecuado cuando se trata con datos categóricos —escalas Likert, por ejemplo- (Flora \& Curran, 2004). Específicamente, en este trabajo se consideraron dos modelos: uno unidimensional (Modelo 1), con una variable latente y 14 ítems como indicadores $(\mathrm{n}=259)$, y uno bidimensional (Modelo 2), con dos variables latentes (CFC-I y CFC-F) y siete ítems por factor $(\mathrm{n}=256)$; para evaluar cada modelo se segmentaron los casos de forma aleatoria.

Posteriormente, para evaluar el ajuste se utilizaron los estadísticos chi-cuadrado, el índice de ajuste comparativo (CFI), el índice de Tucker-Lewis (TLI), el error cuadrático medio de aproximación (RMSEA) y la ponderada media cuadrática residual (WRMR). Aquí, valores superiores a .90 para el CFI y TLI fueron considerados como ajustes aceptables a excelentes; mientras que se esperaron valores entre .05 y .08 para el RMSEA, así como valores menores a 1.00 para la WRMR (Yu \& Muthén, 2002).

Por otra parte, para el estudio de consistencia interna, y considerando las limitaciones del estadístico alfa de Cronbach respecto a su dependencia del número de ítems y las correlaciones entre ellas (Raykov, 2012), se implementó la confiabilidad compuesta $(\rho)$, donde valores iguales o superiores a $\rho=.70$ fueron considerados como aceptables (Nunnally, 1978).

También, para evaluar la validez convergente de la escala, se estudiaron las correlaciones entre los dos factores que componen la CFC (inmediatas y futuras) y las subescalas de la UPPS-P. Para esto, se utilizó el coeficiente de correlación $\mathrm{r}$ de Pearson y se fijó el nivel de significación en $\mathrm{p}<.05$. Finalmente, se evaluó si varones y mujeres reportaban distintos niveles de CFC inmediatas y futuras mediante la realización de un ANOVA univariado, y si había relación entre la edad de los participantes y la puntuación en la escala.

Por último, se analizó la invarianza factorial en función del sexo y la edad de los participantes. Para ello, se realizó un AFC multigrupo con el estimador WLSMV, y se probaron tres niveles de invarianza: configural, métrica y escalar. Para comparar los modelos se empleó el criterio $\Delta \chi^{2}$, aunque - cuando fue necesario - también se consideró el criterio $\Delta \mathrm{CFI} / \Delta \mathrm{TFI}>.01 \mathrm{y}$ el $\Delta \mathrm{RMSEA}>.015$.

\section{Resultados}

Esta sección contiene los resultados de los distintos análisis de datos que se llevaron a cabo. Inicialmente, se presenta el procedimiento realizado con el fin de preparar los datos para los análisis siguientes; luego, se presentan los resultados del AFC, en donde se muestran los valores obtenidos en los distintos índices de bondad de ajuste y de separación de las subescalas; después, se muestran los índices de consistencia interna y los análisis de validez convergente; $y$, finalmente, se presentan los resultados de los análisis de invarianza factorial realizados.

\section{Preparación de los datos}

Inicialmente, los valores faltantes para las respuestas de los ítems de la CFC-14 oscilaron entre $0.4 \%$ (ítem 2) y $2 \%$ (ítem 13), y, debido a que estos no excedieron el $5 \%$ (Schafer, 1999), se procedió a reemplazarlos con la respuesta más frecuente dentro de cada ítem. Cabe resaltar que no hubo valores atípicos univariados $(\mathrm{z}> \pm 3.29)$, pero sí se identificaron 17 casos como valores atípicos multivariados; sin embargo, aunque la presencia de casos atípicos puede distorsionar algunos resultados, se decidió retenerlos para favorecer la generalización (Hair, Anderson, Tatham \& Black, 1999). En la Tabla 1 se muestra la media, la desviación estándar, la asimetría y la curtosis de cada ítem, y allí, es importante resaltar que, sobre la asimetría y la curtosis, 12 ítems mostraron valores de \pm 1 , mientras que dos de ellos mostraron valores por debajo de \pm 2 ; y que las puntuaciones de las subescalas se calcularon utilizando la suma de los ítems de los factores respectivos dividida por el número de ítems por factor, método que elimina las diferencias entre las subescalas debido al número desigual de elementos en cada una (Vásquez-Echeverría et al., 2017).

Seguidamente, los datos faltantes en el UPPS-P variaron de $14.3 \%$-ítem 1 , por ejemplo-a $14.8 \%$-ítem 58 , por ejemplo-, y, teniendo en cuenta que estos valores excedieron el umbral del $5 \%$ (Tabachnick \& Fidell, 2007), el patrón de valores faltantes se verificó mediante la prueba de Little. Con este método, los resultados mostraron que los valores faltantes seguían un patrón aleatorio $\operatorname{MCAR}(\chi 2=293.819$; $\mathrm{df}=348 ; \mathrm{p}=.984)$, razón por la cual los datos faltantes se imputaron al sustituirlos por el modo estadístico. Así, los 37 casos atípicos univariados y los 78 multivariados fueron retenidos (Hair et al., 1999). Ahora bien, con respecto a la asimetría y la curtosis, 47 ítems presentaron valores de \pm 1.00 , considerados excelentes, 11 ítems mostraron valores de \pm 2 , y un solo ítem presentó un valor $> \pm 2$ (George \& Mallery, 2011); los puntajes de cada subescala se calcularon según lo indicado por Verdejo-García et al. (2010).

\section{Análisis factorial confirmatorio}

En general, los resultados para el Modelo 1 (factor único) no indicaron un ajuste adecuado a los datos en los estadísticos empleados $(\mathrm{CFI}=.761 ; \mathrm{TLI}=.718 ; \mathrm{RMSEA}=.146$; IC $90 \%=.138-.155$; WRMR $=2.125)$, y sus estimadores de regresión estandarizados $(\mathrm{p} \leq .05)$ variaron de .19 a .81 . Mientras que el Modelo 2 se ajustó ligeramente mejor que 
Tabla 1.

Estadísticos descriptivos de la escala CFC-14 (versión en español)

1. I consider how things might be in the future, and try to influence those things
with my day-to-day behavior (Considero cómo serán los eventos en el futuro y trato de influenciar esos eventos con mi comportamiento diario), $\mathrm{F}$.

\section{Media}

2. Often I engage in a particular behavior in order to achieve outcomes that may not result for many years (A menudo me comporto de una manera particular para obtener resultados que no se verán hasta pasados muchos años), $\mathrm{F}$.

3. I only act to satisfy immediate concerns, figuring the future will take care of itself (Solo actúo para satisfacer mis preocupaciones inmediatas, pienso que en el futuro se resolverán solas), I.

4. My behavior is only influenced by the immediate (i.e., a matter of days or weeks) outcomes of my actions (Mi comportamiento solo es influenciado por los resultados inmediatos de mis acciones (por ejemplo: un problema de días o semanas), I.

5. My convenience is a big factor in the decisions I make or the actions I take (Mi beneficio es un factor importante en las decisiones que tomo o en las acciones que realizo), I.

6. I am willing to sacrifice my immediate happiness or wellbeing in order to achieve future outcomes (Estoy dispuesto/a a sacrificar mi felicidad o bienestar inmediato para lograr resultados futuros), $\mathrm{F}$.

7. I think it is important to take warnings about negative outcomes seriously even if the negative outcome will not occur for many years (Pienso que es importante tener precaución sobre los resultados negativos aún si no ocurrirán por muchos años), F.

8. I think it is more important to perform a behavior with important distant consequences than a behavior with less important immediate consequences (Creo que es más importante realizar algo que tendrá importantes consecuencias a largo plazo que un comportamiento con consecuencias inmediatas menos importantes), F.

9. I generally ignore warnings about possible future problems because I think the problems will be resolved before they reach crisis level (Generalmente ignoro advertencias sobre posibles problemas futuros porque pienso que los problemas se resolverán antes de llegar a un nivel crítico), I.

10. I think that sacrificing now is usually unnecessary since future outcomes can be dealt with at a later time (Pienso que sacrificarse ahora es generalmente innecesario ya que situaciones futuras podrán ser resueltas más adelante), I.

11. I only act to satisfy immediate concerns, figuring that I will take care of future problems that may occur at a later date (Solo actúo para satisfacer preocupaciones inmediatas, calculando que más adelante me preocuparé de problemas futuros que puedan ocurrir), I.

12. Since my day-to-day work has specific outcomes, it is more important to me than behavior that has distant outcomes (Dado que mi trabajo cotidiano tiene resultados especificos, para mí eso es más importante que la conducta que tiene resultados distantes), I.

13. When I make a decision, I think about how it might affect me in the future (Cuando tomo una decisión, pienso en cómo podría afectarme en el futuro), $\mathrm{F}$.

14. My behavior is generally influenced by future consequences (Mi comportamiento generalmente es influenciado por las consecuencias futuras), F.

3.92

$\begin{array}{llll}4.81 & 1.67 & -0.52 & -0.33\end{array}$

0.96

Nota. $\mathrm{F}=$ futuro; $\mathrm{I}=$ inmediato.

\begin{tabular}{llll}
2.86 & 1.63 & 0.63 & -0.27 \\
5.63 & 1.56 & -1.17 & 0.79 \\
4.72 & 1.61 & -0.51 & -0.28 \\
\hline
\end{tabular}


el Modelo 1, pues logró un ajuste satisfactorio en los mismos estadísticos $(\mathrm{CFI}=.930 ; \mathrm{TLI}=.916 ; \mathrm{RMSEA}=.080$; IC $90 \%=.071-.089 ;$ WRMR $=1.235)$, y sus estimadores de regresión estandarizados $(\mathrm{p} \leq .05)$ variaron entre $.49 \mathrm{y}$ .75 para CFC-F y entre 10 y .83 para CFC-I.

En particular, en el Modelo 2 el ítem 5 tuvo una carga factorial baja (.10), por lo que se procedió a excluirlo y volver a calcular el modelo $(\mathrm{CFI}=.950 ; \mathrm{TLI}=.939$; RMSEA $=.071$; IC $90 \%=.063-.082$; WRMR $=1.086$ ). Asimismo, debido a que los ítems 13 y 14 estaban altamente correlacionados, se decidió intercorrelacionarlos $\mathrm{y}$ reevaluar el ajuste del modelo $(\mathrm{CFI}=.961 ; \mathrm{TLI}=.952$; RMSEA $=.064$; IC $90 \%=.054-.074$; WRMR $=.979), \mathrm{y}$ así, los estimadores de regresión estandarizados $(\mathrm{p} \leq .05)$ variaron entre .50 y .66 en la CFC-F, y entre .43 y .83 para la CFC-I (véase Figura 1 para una inspección de los estimadores beta estandarizados). Estos resultados se resumen en la Tabla 2.

Es importante señalar que, aunque en la presentación de los datos a continuación se utiliza la etiqueta "CFC-14", en realidad los análisis que se presentan se realizaron utilizando la versión de 13 ítems, sin el ítem 5.

\section{Consistencia interna}

Los valores de fiabilidad compuestos fueron satisfactorios para los diferentes modelos analizados. En particular, al considerar una medida general de la escala $\mathrm{CFC}$ - todos los ítems combinados después de que los ítems inmediatos fueron recodificados-, se logró un valor $\rho=.84$ (Modelo 1 ); mientras que, cuando ambos factores se consideraron por separado (Modelo 2), la subescala CFC-F logró un valor $\rho=.81$ y la subescala CFC-I mostró un valor $\rho=.79$; , cuando se eliminó el ítem cinco de la subescala CFC-I, el CFC-F logró un $\rho=.80$ y el CFC-I, un $\rho=.82$ (véase Tabla 2).

\section{Validez convergente}

Por otra parte, como se muestra en la Tabla 3, se encontraron correlaciones significativas y positivas entre la subescala CFC-I y todas las subescalas UPPS-P, excepto en

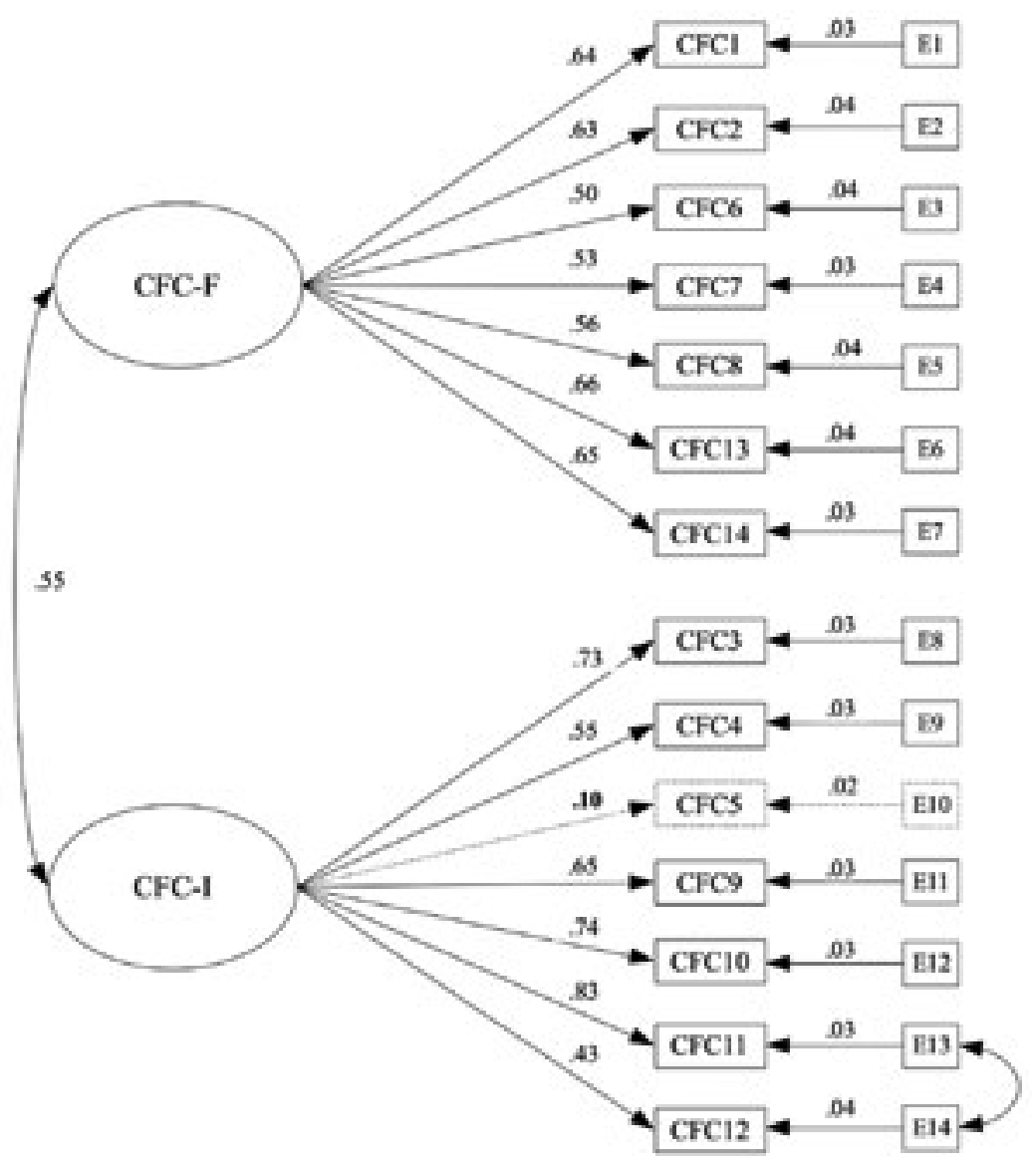

Figura 1. Análisis factorial confirmatorio de la escala CFC de 13 y 14 ítems. Todas las vías fueron significativas $(\mathrm{p}<.05)$ y todos los ítems cargaron en su factor esperado, excepto en la vía de línea de puntos (CFC5). 
Tabla 2.

Fiabilidad compuesta ( $\rho$ ) e indices de ajuste para los modelos alternativos del CFC-14

\begin{tabular}{|c|c|c|c|c|c|c|c|}
\hline Modelo & $\rho$ CFC-F/CFC-I & $\chi^{2}$ & $\mathrm{df}$ & CFI & TLI & RMSEA (IC $90 \%$ ) & WRMR \\
\hline Un factor & .84 & $918.093 *$ & 77 & .761 & .718 & $.146(.138 / .155)$ & 2.125 \\
\hline Dos factores (14 ítems) & $.81 / 79$ & $322.291 *$ & 76 & .930 & .916 & $.080(.071 / .089)$ & 1.235 \\
\hline Dos factores (13 ítems) & $.80 / .82$ & $195.678 *$ & 63 & .961 & .952 & $.064(.054 / .074)$ & 0.979 \\
\hline
\end{tabular}

Nota . CFI = índice de ajuste comparativo; TLI = índice Tucker-Lewis; RMSEA = aproximación del error cuadrado de la media; $\mathrm{IC}=$ intervalo de confianza; WRMR = media ponderada residual cuadrática. ${ }^{*} p<.001$.

la búsqueda de sensaciones, que no fue significativa; mientras que entre las subescalas CFC-F y las de la UPPS-P, todas las correlaciones fueron negativas y significativas, donde destacó la correlación negativa y moderada entre la CFC-F y la falta de premeditación, una dimensión estrictamente relacionada con la toma de decisiones intertemporales.

Además, se analizó la relación entre las puntuaciones en diferentes subescalas y la edad, pero no se encontraron relaciones significativas. Del mismo modo, no se encontraron diferencias entre los géneros en las puntuaciones en ambas subescalas.

\section{Invariancia factorial}

Invariancia según el sexo. Inicialmente no fue posible estimar la invariancia utilizando la escala de respuesta original de siete puntos, debido a que en algunos grupos la frecuencia en las opciones extremas era cercana o igual a cero, lo que resulta incompatible con el estimador WLSMV. En consecuencia, se tuvo que re-escalar las opciones de respuesta de siete a cinco puntos (las categorías 1 y 2 y 6 y 7 se colapsaron).

De este modo, el CFA-GM se llevó a cabo con la escala de cinco puntos, y, como resultados, primero, el modelo de configuración mostró un ajuste aceptable $\left(\chi^{2}=295.026\right.$; $\mathrm{df}=130 ; \mathrm{p}=.000 ; \mathrm{CFI}=.930 ; \mathrm{TLI}=.916 ; \mathrm{RMSEA}=.071$; IC $90 \%=.060-.081$; WRMR $=1.407$ ); en segundo lugar, cuando se probó el modelo métrico, se observó un excelente ajuste $\left(\chi^{2}=258.892 ; \mathrm{df}=141 ; \mathrm{p}=.000\right.$; $\mathrm{CFI}=.950 ; \mathrm{TLI}=.945 ; \mathrm{RMSEA}=.057$; IC $90 \%=.046-$ $.068 ;$ WRMR $=1.430$ ); el modelo escalar también mostró un ajuste excelente $\left(\chi^{2}=298.161 ; \mathrm{df}=193 ; \mathrm{p}=.000\right.$; $\mathrm{CFI}=.955 ; \mathrm{TLI}=.964 ; \mathrm{RMSEA}=.046 ; \mathrm{IC} 90 \%=.036-.056$;
WRMR = 1.593), de manera similar al modelo métrico; $y$, además, el modelo escalar no fue significativamente diferente del modelo métrico $\left(\Delta \chi^{2}=58.759 ; \mathrm{df}=52, \mathrm{p}=.2417\right)$. En consecuencia, resulta posible suponer que existe invariancia entre los grupos en función del sexo.

Invariancia según la edad. Para analizar la invariancia por edad, la muestra se dividió en dos grupos, uno de jóvenes - de 18 a 25 años - y otro de adultos — de 26 a 60 añosLuego, el modelo de configuración mostró un ajuste aceptable $\left(\chi^{2}=266.613 ; \mathrm{df}=130 ; \mathrm{p}=.000 ; \mathrm{CFI}=.941 ; \mathrm{TLI}=.929\right.$; RMSEA $=.064$; IC $90 \%=.053-.075$; WRMR $=1.287$ ); el modelo métrico mostró excelentes valores de ajuste $\left(\chi^{2}=245.424 ; \mathrm{df}=141 ; \mathrm{p}=.000 ; \mathrm{CFI}=.955 ; \mathrm{TLI}=.950\right.$; RMSEA $=.055$; IC $90 \%=.043-.065$; WRMR $=1.364$ ), que no se diferenciaron significativamente del modelo de configuración $\left(\Delta \chi^{2}=11.768 ; \mathrm{df}=11, \mathrm{p}=.3813\right)$; pero, finalmente, el modelo escalar se ajustó aún mejor $\left(\chi^{2}=286.126\right.$; $\mathrm{df}=193 ; \mathrm{p}=.000 ; \mathrm{CFI}=.960 ; \mathrm{TLI}=.967 ; \mathrm{RMSEA}=.044$; IC $90 \%=.032-.054$; WRMR $=1.537$ ), aunque no se diferenció significativamente del modelo métrico $\left(\Delta \chi^{2}=59.704\right.$; $\mathrm{df}=52, \mathrm{p}=.2160)$. En consecuencia, es posible suponer que existe invariancia según la edad.

\section{Discusión}

Las decisiones cotidianas de las personas, sean triviales o más importantes, tienen un efecto en su futuro cercano o distante, sin embargo, suelen haber diferencias en cuanto a la forma en que el considerar las consecuencias de sus decisiones afecta la actividad misma de tomarlas. Esta característica distintiva se denomina consideración de las

Tabla 3.

Matriz de correlación entre las subescalas de la CFC-14 y la escala UPPS-P

\begin{tabular}{lccccc}
\hline & $\begin{array}{c}\text { Urgencia } \\
\text { negativa }\end{array}$ & Falta de premeditación & Falta de perseverancia & $\begin{array}{c}\text { Búsqueda de } \\
\text { sensaciones }\end{array}$ & Urgencia positiva \\
\hline CFC-I & $.22^{* *}$ & $.18^{* *}$ & $.26^{* *}$ & .07 & $.25^{* *}$ \\
CFC-F & $-.09^{*}$ & $-.41^{* *}$ & $-.29^{* *}$ & $-.13^{* *}$ & $-.09^{*}$ \\
\hline
\end{tabular}

Nota. $\mathrm{N}=512 . * \mathrm{p} \leq .05 * * \mathrm{p}<.01$. 
consecuencias futuras (CFC), y se define como la medida en que las personas consideran y están influenciadas por las posibles consecuencias futuras de su comportamiento actual (Nigro et al., 2016; Strathman et al., 1994).

Teniendo en cuenta que hay una escala validada para medir este constructo, la escala de Consideración de Consecuencias Futuras (CFC-14) — versión de 14 ítems, el objetivo de este trabajo fue traducir y adaptar dicho instrumento a una población general de Córdoba, Argentina. En particular, esta meta se vinculó a dos objetivos de trabajo: el primero, aportar un nuevo elemento al conjunto de instrumentos disponibles en Argentina para evaluar este constructo, ya que, a pesar de que hay varias adaptaciones de la CFC-14 a otros países, como lo recomienda la literatura (Carretero-Dios \& Pérez, 2007; Pilatti, Godoy \& Brussino, 2012), resulta conveniente hacer las adaptaciones necesarias para saber cómo funciona un instrumento creado en una cultura diferente; y el segundo, evaluar adecuadamente cómo las personas toman sus decisiones sobre la salud, el medio ambiente y el comportamiento riesgoso. Así, con este trabajo se inicia una de las primeras etapas de un proyecto más amplio, destinado a desarrollar un modelo de ecuación estructural sobre cómo y por qué los adolescentes y los jóvenes toman decisiones riesgosas en su vida cotidiana, considerando factores personales y ambientales.

Ahora bien, contrario a los primeros reportes para la versión original de CFC de 12 ítems (Strathman et al., 1994), en distintos estudios (Joireman et al., 2008; Nigro et al., 2016), y en el presente trabajo de investigación, se encontró que el modelo de un factor no se ajusta adecuadamente a los datos, y que la estructura interna de la CFC-14 se ajusta mejor a un modelo de dos factores, uno que considera las consecuencias inmediatas (CFC-I, siete ítems) y otro que considera las consecuencias futuras (CFC-F, siete ítems), tal como lo propuso Joireman et al. (2012).

Sin embargo, aquí se encontró que el ítem 5 ("mi conveniencia es un factor importante en las decisiones que tomo o en las acciones que tomo"), perteneciente al factor CFC-I, no aportó una contribución significativa; hallazgo similar a lo encontrado por Vásquez-Echeverría et al. (2017) y Vásquez-Echeverría et al. (2015) respecto a este ítem. Al respecto, se puede encontrar una explicación a esto en la versión original de la escala, ya que la redacción del ítem 5 se refiere al término "conveniencia", que es difícil de traducir y adaptar a los idiomas basados en el latín debido a su ambigüedad. Por lo tanto, siguiendo las recomendaciones de Vásquez Echeverría et al. (2017), en el presente estudio se procedió a eliminar y reevaluar la estructura factorial, lo que permitió una ligera mejora en los índices de ajuste.

Por otra parte, la validez convergente entre la CFC y la impulsividad se examinó mediante la comparación de los resultados de este instrumento con los de la Escala de Impulsividad (UPPS-P) de Verdejo-García et al. (2010). En general, esta escala consta de cinco dimensiones, dos relacionadas con la consideración de la temporalidad subjetiva: la falta de premeditación — la más estrictamente relacionada con la dimensión temporal-, que se define como la tendencia a actuar sin considerar las consecuencias del comportamiento actual —es decir, iniciar un proyecto sin saber cómo proceder-; y la falta de perseverancia, definida como la dificultad de permanecer en una tarea que puede volverse difícil o aburrida (Gagnon, Daelman, Mcduff \& Kocka, 2013).

Si bien la falta de perseverancia se asocia más a menudo con problemas de atención, la falta de persistencia en una tarea que tiene beneficios a largo plazo — es decir, estudiar para un examen o seguir una dieta baja en grasas-, pues podría implicar no considerar las consecuencias futuras del comportamiento actual y enfocarse solo en las consecuencias más cercanas en el tiempo (Nigro et al., 2016). Ahora, los resultados encontrados en la correlación entre los puntajes en la CFC-F y la falta de premeditación y las dimensiones de falta de perseverancia respaldan la idea de que estos constructos están asociadas, por lo que se puede afirmar que quienes no premeditan sus acciones y quienes no perseveran en ellas, a su vez, no muestran puntajes altos en la subescala CFC-F.

Con respecto a la relación entre la edad y los puntajes CFC-14, en este trabajo no se encontró una correlación estadísticamente significativa. Es posible que la ausencia de hallazgos significativos en este estudio y en el de VásquezEcheverría et al. (2015) se deba al hecho de que todos los participantes en ambos estudios eran mayores de 18 años, pues en el estudio de Vásquez-Echeverría et al. (2015) la edad y el puntaje de CFC correlacionaron solo cuando se consideraron participantes de 13 años en adelante. Del mismo modo, otros estudios han encontrado una asociación significativa entre la edad y el CFC, con en el de Nigro et al. (2016), donde se encontró una correlación negativa moderada entre la edad y las puntuaciones generales (14 ítems) en una submuestra mayor de participantes de 16 a 19 años; además de que, cuando analizaron las puntuaciones en cada subescala, los adultos obtuvieron puntuaciones más altas que los adolescentes en la subescala CFC-F.

Ahora bien, sobre la validez externa de este estudio, se puede mencionar, primero, que la proporción desigual de género en la muestra, con más mujeres que hombres, socava la importancia de los hallazgos; $y$, segundo, que el nivel educativo de los participantes no era heterogéneo, ya que quienes tenían escolarización secundaria completa e incompleta eran muy pocos en relación con la muestra completa, en su mayoría compuesta por personas con nivel 
366

educativo terciario/universitario o superior. Estos aspectos, sumados al modo de administración de la escala, pudieron haber afectado la representatividad de estos resultados para la población en general - aunque el hecho de que no todos los participantes fueran estudiantes universitarios favorece la generalización de los resultados-. Además, haber comparado diferentes soluciones de factores proporciona evidencia de que el modelo de dos factores es el más adecuado para la población general a nivel local, lo que también coincide con los resultados de otros estudios (Nigro et al., 2016).

Por otro lado, los análisis progresivos de invariancia factorial muestran que, a pesar de los problemas reportados para formar una muestra con grupos equilibrados en sexo y edad, es posible comparar la puntuación entre los grupos de edad y sexo porque se puede sostener que existe una invariancia factorial progresiva entre los diferentes grupos de la muestra - la invariancia factorial es importante cuando se comparan los grupos en función del sexo, la edad y la cultura - Esto significa que las puntuaciones obtenidas por cada grupo son comparables entre sí, porque representan lo mismo y las diferencias se pueden interpretar como diferencias en las características y no atribuibles a fuentes desconocidas (Domínguez-Lara \& Medrano, 2016).

Es importante señalar que los resultados de este estudio son solo los primeros derivados de una investigación más amplia dirigida a evaluar las características de la CFC y de los instrumentos disponibles para su medición. Al respecto, se considera necesario avanzar en la evaluación de la convergencia entre los puntajes de la $\mathrm{CFC}$ y las pruebas experimentales de la perspectiva del tiempo, conceptualizada como "el proceso no consciente por el cual los flujos continuos de experiencias personales y sociales se asignan a categorías temporales, o marcos de tiempo, que ayuda para dar orden, coherencia y significado a esos eventos" (Zimbardo \& Boyd, 2015). Del mismo modo, es necesario evaluar la estabilidad de los puntajes mediante diseños testretest (Nigro et al., 2016) y analizar las diferencias en la $\mathrm{CFC}$ en muestras con diferentes rasgos de personalidad y características como la edad, intereses, ideología política, entre otros, en la población local (Bruderer-Enzler, 2015; Gick, 2014; Joireman \& King, 2016; Joireman et al., 2012).

Es preciso afirmar que este trabajo ofrece evidencia sobre la validez de la escala de Consideración de Consecuencias Futuras (CFC-14), por lo cual se concluye que esta escala permite una evaluación apropiada del constructo en una población local. En este sentido, si se presta atención a cómo piensan las personas —adolescentes, jóvenes y adultosy cómo su contexto y condiciones históricas dan forma a las condiciones de pensamiento y toma de decisiones, con la información adecuada es posible mejorar el diseño y la implementación de intervenciones y políticas centradas en la elección y la decisión (Banco Mundial, 2015; Joireman \& King, 2016). Por lo tanto, los instrumentos que amplían el conocimiento sobre cómo varía la toma de decisiones entre grupos y contextos específicos ayudan a mejorar el diseño y el impacto de las intervenciones de comportamiento de salud dirigidas a mejorar la calidad de vida de poblaciones específicas.

En síntesis, la consideración de las consecuencias futuras (CFC) es un rasgo de personalidad definido como el grado en que las personas anticipan y están influenciadas por las posibles consecuencias inmediatas y futuras de su comportamiento actual (Joireman \& King, 2016; Strathman et al., 1994), y, por tanto, medirlo con precisión resulta importante para desarrollar intervenciones apropiadas con respecto a la salud y el bienestar de las personas, así como considerar cómo las diferencias personales en los valores políticos, ambientales y sociales pueden dar forma a los diferentes tipos de comportamiento presente y futuro. Así, una traducción argentina del CFC-14 (Joireman et al., 2012) es útil debido a las diferencias de idioma con otras traducciones al español (Vásquez-Echeverría et al., 2017) ya disponibles.

Por último, se concluye que los resultados presentados aquí muestran que la versión argentina de CFC-14 tiene propiedades psicométricas adecuadas en una muestra general: los ítems cargaron en dos factores separados, uno con respecto a las consecuencias más distantes (CFC-Futuro) y otro con respecto a las consecuencias más cercanas (CFCInmediato); el ítem cinco mostró problemas y se abandonó, aspecto que ya había ocurrido con otras traducciones (Vásquez-Echeverría et al., 2015, 2017) y puede deberse a la redacción en español; el estudio de validez discriminante muestra que la CFC es una construcción diferente de la impulsividad — medida con la Escala de Impulsividad (UPPS-P), instrumento con excelentes propiedades psicométricas en la población argentina (Pilatti et al., 2015)-; y los resultados de la invariancia factorial evidencian que es posible comparar las puntuaciones en diferentes grupos, al menos con respecto a la edad y el sexo, hallazgo clave porque permite continuar usando este instrumento como parte de una batería más grande y con la intención de establecer diferencias en el procesamiento del tiempo en diferentes poblaciones. Por lo tanto, es posible afirmar que la versión argentina de CFC-14 es un instrumento apropiado para evaluar la consideración del rasgo de consecuencias futuras en la población local. 


\section{Referencias}

Adams, J. (2012). Consideration of immediate and future consequences, smoking status, and body mass index. Health Psychology, 31(2), 260-263. doi: https://doi.org/10.1037/ a0025790

Azizli, N., Atkinson, B. E., Baughman, H. M., \& Giammarco, E. A. (2015). Relationships between general self-efficacy, planning for the future, and life satisfaction. Personality and Individual Differences, 82, 58-60. doi: https://doi. org/10.1016/j.paid.2015.03.006

Banco Mundial. (2015). Mente, sociedad y conducta. Washington, D. C. doi: https://doi.org/10.1787/ leo-2014-6-es

Bruderer-Enzler, H. (2015). Consideration of future consequences as a predictor of environmentally responsible behavior: Evidence from a general population study. Environment and Behavior, 47(6), 618-643. doi: https://doi. org/10.1177/0013916513512204

Camus, G., Berjot, S., \& Ernst-Vintila, A. (2014). Validation française de l'échelle de prise en considération des conséquences futures de nos actes (CFC-14). Revue Internationale de Psychologie Sociale, 27(1), 35-63. Recuperado de https:// www.cairn.info/revue-internationale-de-psychologie-socia le-2014-1-page-35.htm

Carretero-Dios, H., \& Pérez, C. (2007). Standards for the development and review of instrumental studies: Considerations about test selection in psychological research. International Journal of Clinical and Health Psychology, 7(3), 863-882. Recuperado de https://www.redalyc.org/pdf/337/33770319.pdf

Dominguez-Lara, S. A., \& Medrano, L. A. (2016). Invarianza factorial del Cognitive Emotional Regulation Questionnarie (CERQ) en universitarios limeños y cordobeses. Universitas Psychologica, 15(1), 89-98. doi: https://doi.org/10.11144/ Javeriana.upsy 15 -1.ifce

Flora, D. B., \& Curran, P. J. (2004). An empirical evaluation of alternative methods of estimation for confirmatory factor analysis with ordinal data. Psychological Methods, 9(4), 466-491. doi: https://doi.org/2004-21445-005[pii]\ r10.1037/1082-989X.9.4.466

Gagnon, J., Daelman, S., Mcduff, P., \& Kocka, A. (2013). UPPS Dimensions of Impulsivity Relationships with Cognitive Distortions and Childhood Maltreatment. Journal of Individual Differences, 34(1), 48-55. doi: https:// doi.org/10.1027/1614-0001/a000099

Geers, A. L., Wellman, J. A., Seligman, L. D., Wuyek, L. A., \& Neff, L. A. (2010). Dispositional optimism, goals, and engagement in health treatment programs. Journal of Behavioral Medicine, 33(2), 123-134. doi: https://doi. org/10.1007/s10865-009-9238-z

George, M., \& Mallery, P. (2011). Descriptive Statistics. En S. Hartman (ed.), SPSS for Windows step by step. A simple guide and reference 18.0 update (pp. 95-104). Boston: Pearson.
Gick, M. (2014). An exploration of interactions between Conscientiousness and Consideration of Future Consequences on healthy eating. Personality and Individual Differences, 66, 181-187. doi: https://doi.org/10.1016/j. paid.2014.03.020

Hair, J., Anderson, R., Tatham, R., \& Black, W. (1999). Análisis Multivariante (5. ${ }^{\mathrm{a}}$ ed.). Madrid: Prentice Hall.

Joireman, J., Balliet, D., Sprott, D., Spangenberg, E., \& Schultz, J. (2008). Consideration of future consequences, ego-depletion, and self-control: Support for distinguishing between CFC-Immediate and CFC-Future sub-scales. Personality and Individual Differences, 45(1), 15-21. doi: https://doi.org/10.1016/j.paid.2008.02.011

Joireman, J., \& King, S. (2016). Individual Differences in the Consideration of Future and (More) Immediate Consequences: A Review and Directions for Future Research. Social and Personality Psychology Compass, 5(5), 313-326. doi: https://doi.org/10.1111/spc3.12252

Joireman, J., Shaffer, M. J., Balliet, D., \& Strathman, A. (2012). Promotion Orientation Explains Why Future-Oriented People Exercise and Eat Healthy: Evidence from the TwoFactor Consideration of Future Consequences-14 Scale. Personality and Social Psychology Bulletin, 38(10), 12721287. doi: https://doi.org/10.1177/0146167212449362

Joireman, J., Strathman, A., \& Balliet, D. (2006). Considering future consequences. En L. Sanna \& E. Chang (eds.), Judgments Over Time: The Interplay of Thoughts, Feelings, and Behaviors (pp. 82-99). Oxford: Oxford University Press. doi: https://doi.org/10.1093/acprof

Mimura, C., \& Griffiths, P. (2008). A Japanese version of the Rosenberg Self-Esteem Scale: Translation and equivalence assessment. BMC Psychiatry, 8. doi: https://doi. org/10.1016/j.jpsychores.2006.11.004

Montero, I., \& León, O. G. (2007). A guide for naming research studies in Psychology. International Journal of Clinical and Health Psychology, 7(3), 847-862. Recuperado de https:// www.redalyc.org/pdf/337/33770318.pdf

Nigro, G., Cosenza, M., Ciccarelli, M., \& Joireman, J. (2016). An Italian translation and validation of the Consideration of Future Consequences-14 Scale. Personality and Individual Differences, 101, 333-340. doi: https://doi.org/10.1016/j. paid.2016.06.014

Nunnally, J. C. (1978). Psychometric theory (2. ${ }^{\text {a }}$ ed.). New York: McGraw-Hill.

Petrocelli, J. V. (2003). Factor validation of the Consideration of Future Consequences Scale: evidence for a short version. The Journal of Social Psychology, 143(4), 405-413. doi: https://doi.org/10.1080/00224540309598453

Pilatti, A., Godoy, J. C., \& Brussino, S. A. (2012). Adaptación de instrumentos entre culturas: ejemplos de procedimientos seguidos para medir las expectativas hacia el alcohol en el ámbito argentino. Trastornos Adictivos, 14(2), 58-64. doi: https://doi.org/10.1016/S1575-0973(12)70045-4 
Pilatti, A., Lozano, O., \& Cyders, M. (2015). Psychometric Properties of the Spanish Version of the UPPS-P Impulsive Behavior Scale: A Rasch Rating Scale Analysis and Confirmatory Factor Analysis. Psychological Assessment, 27(4), 10-21. doi: 10.1037/pas0000124

Pilatti, A., Rivarola-Montejano, G., Lozano, O. M., \& Pautassi, R. M. (2016). Relationship between impulsivity and alcohol consumption in Argentinean men and women. Quaderns de Psicologia, 18(1), 75. doi: https://doi.org/10.5565/rev/ qpsicologia.1329

Raykov, T. (2012). Evaluation of latent construct correlations in the presence of missing data: a note on a latent variable modelling approach. The British Journal of Mathematical and Statistical Psychology, 65(1), 19-31. doi: https://doi. org/10.1348/000711010X498162

Schafer, J. (1999). Multiple imputation: a primer. Statistical Methods in Medical Research, 8, 3-15. doi: https://doi. org/10.1191/096228099671525676

Strathman, A., Gleicher, F., Boninger, D. S., \& Edwards, C. S. (1994). The consideration of future consequences: Weighing immediate and distant outcomes of behavior. Journal Personality and Social Psychology, 66(4), 742752. doi: https://doi.org/10.1037/0022-3514.66.4.742

Tabachnick, B., \& Fidell, L. (2007). Using Multivariate Statistics (5. ${ }^{\mathrm{a}}$ ed.). Boston: Pearson Education.

Toepoel, V. (2010). Is consideration of future consequences a changeable construct? Personality and Individual Differences, 48(8), 951-956. doi: https://doi.org/10.1016/j. paid.2010.02.029
Vásquez-Echeverría, A., Esteves, C., Vilares-Gomes, C., Ortuño, V., Gomes, C., \& Ortuño, V. (2015). Portuguese Validation of the Consideration of Future Consequences Scale. The Spanish Journal of Psychology, 18, 1-11. doi: https://doi.org/10.1017/sjp.2015.5

Vásquez-Echeverría, A., Martín, A., Esteves, C., Ortuño, V. E. C., \& Joireman, J. (2017). Adaptación y Validación Inicial al Castellano de la Escala de Consideración de las Consecuencias Futuras. Revista Iberoamericana de Diagnóstico y Evaluación Psicológica, 44(2), 5-15. doi: 10.21865/RIDEP44.2.01

Verdejo-García, A., Lozano, Ó., Moya, M., Alcázar, M. Á., \& Pérez-García, M. (2010). Psychometric properties of a Spanish version of the UPPS-P impulsive behavior scale: reliability, validity and association with trait and cognitive impulsivity. Journal of Personality Assessment, 92(1), 7077. doi: https://doi.org/10.1080/00223890903382369

Yu, C., \& Muthen, B. (2002). Evaluation of model fit indices for latent variable models with categorical and continuous outcomes. Paper presented at the annual conference of the American Educational Research Association. New Orleans, LA.

Zimbardo, P., \& Boyd, J. (2015). Time perspective theory; Review, research and application: Essays in honor of Philip G. Zimbardo. En M. Stolarski, N. Fieulaine \& W. van Beek (eds.), Time Perspective Theory; Review, Research and Application: Essays in Honor of Philip G. Zimbardo (pp. 1755). Cham: Springer International Publishing Switzerland. doi: https://doi.org/10.1007/978-3-319-07368-2 